\title{
New examples of static spacetimes admitting a unique standard decomposition
}

\author{
Alma L. Albujer • Jónatan Herrera . \\ Rafael M. Rubio
}

Received: date / Accepted: date

\begin{abstract}
In this paper we introduce a new general approach for the study of spacetimes admitting a standard static splitting. This approach allows us to give an alternative proof for the uniqueness of splitting in the spatially closed case to the first study made by Sánchez-Senovilla and later by Aledo-RomeroRubio. However, our technique also allows us to obtain new uniqueness results for standard static models with complete (non necessarily compact) spacelike bases under some mild hypothesis, including some restrictions on the sectional curvature of such bases.
\end{abstract}

Keywords Standard static spacetime - Standard static splitting - Global Killing vector field · Maximal hypersurface

Mathematics Subject Classification (2010) 53C50 - 53C $80 \cdot 83 \mathrm{C} 20$

The first author is partially supported by MINECO/FEDER project reference MTM201565430-P, Spain, and Fundación Séneca project reference 19901/GERM/15, Spain. Her work is a result of the activity developed within the framework of the Program in Support of Excellence Groups of the Región de Murcia, Spain, by Fundación Séneca, Science and Technology Agency of the Región de Murcia. The second author is partially supported by the Spanish Grant MTM2016-78807-C2-2-P (MINECO and FEDER funds). The third author is partially supported by the Spanish MINECO and ERDF project Grant MTM2016-78807$\mathrm{C} 2-1-\mathrm{P}$

\footnotetext{
A. L. Albujer

Departamento de Matemáticas, Edificio Albert Einstein

Universidad de Córdoba, Campus de Rabanales

14071 Córdoba, Spain E-mail: alma.albujer@uco.es

J. Herrera

Departamento de Matemáticas, Edificio Albert Einstein

Universidad de Córdoba, Campus de Rabanales

14071 Córdoba, Spain E-mail: jherrera@uco.es

R. M. Rubio

Departamento de Matemáticas, Edificio Albert Einstein

Universidad de Córdoba, Campus de Rabanales

14071 Córdoba, Spain E-mail: rmrubio@uco.es
} 


\section{Introduction}

A connected, oriented Lorentz manifold $M$ admitting a globally defined timelike vector field is time-oriented, so it is a spacetime [16, Definition 1.3.1]. If in addition, such a vector field is also Killing, the spacetime is said to be stationary. Among all the stationary spacetimes, those for which the Killing vector field is irrotational are known in the literature as static spacetimes. From the physical standpoint, those spaces are static relative to the observer field $Z=\frac{1}{\|K\|} K$ determined by $K$. Let us recall that a vector field $K$ is irrotational if and only if its orthogonal distribution $K^{\perp}$ is integrable, [15, Proposition 12.30]. As a consequence, from the classical Fröbenius theorem, [19, Theorem 1.64], for each event $p \in M$, there exists a connected inextensible spacelike hypersurface $\mathcal{F}_{p}$ in $M$, such that $p \in \mathcal{F}_{p}$ and $T_{q} \mathcal{F}_{p}=K_{q}^{\perp}$, for all $q \in \mathcal{F}_{p}$. In fact, $\mathcal{F}_{p}$ is called the leaf of the foliation $K^{\perp}$ through $p \in M$, and may be interpreted as the spatial universe (restspace) of each observer determined by $K$, which intersects $\mathcal{F}_{p}$ at an instant of its proper time. Just as a remark, along this paper we will understand hypersurfaces, and submanifolds in general, as immersions (or inmersed submanifolds). Therefore, they are not necessarily embeddings in $M$. This point of view corresponds with the definition of submanifold in many classical texts, see for instance [19, Definition 1.27].

In addition, any local flow $\left\{\phi_{t}\right\}$ of the Killing vector field $K$ consists of (local) isometries of $M$ which preserve restspaces of observers determined by $K$. In other words, $\phi_{t}\left(\mathcal{F}_{p}\right)$ is contained in the integral leaf of $K^{\perp}$ through $\phi_{t}(p)$. Therefore, the spatial universe always looks the same for the observers determined by $K$, at least locally. Taking this fact into account, it is not difficult to see that for each $p \in M$ there is a local isometry of a neighbourhood at $p$ in $M$ into a warped product $\left(I_{\alpha} \times \Sigma, g\right)$, in the sense of [15, Chapter 7]. Specifically, the fiber $I \subseteq \mathbb{R}$ is an open interval, the base $\Sigma$ an $n$-dimensional Riemannian manifold $(\Sigma, h)$, the warping function $\alpha$ a positive smooth function $\alpha \in \mathcal{C}^{\infty}(\Sigma)$, and

$$
g=-\alpha\left(\pi_{\Sigma}\right)^{2} \pi_{I}^{*}\left(d t^{2}\right)+\pi_{\Sigma}^{*}(h),
$$

where $\pi_{I}$ and $\pi_{\Sigma}$ denote the projections of $I \times \Sigma$ onto $I$ and $\Sigma$, respectively. Moreover, this isometry preserves the observer field $Z$ determined by the Killing vector field $K$, which is transformed into $\partial_{t}:=\partial / \partial t$, [15, Lemma 12.37].

Furthermore, in the case where the previously described local isometry is, in fact, a global one, the spacetime is called a standard static spacetime. To simplify the notation, from now on we will just identify a standard static spacetime with its warped product representation. Therefore, we will say that a standard static spacetime $(M, g)$ admits a global splitting as in (1), and we will abbreviate it as

$$
M=\mathbb{R} \times \Sigma, \quad g=-\alpha^{2} d t^{2}+h .
$$

In any standard static spacetime there is a remarkable family of spacelike hypersurfaces, namely its spacelike slices or leaves $\Sigma_{t_{0}}=\left\{t_{0}\right\} \times \Sigma, t_{0} \in I$. It is 
easy to check that the shape operator of any spacelike slice vanishes identically, i.e., they are totally geodesic. Moreover, if $(\Sigma, h / \alpha)$ is a complete Riemannian manifold, it is well known that $M$ is then globally hyperbolic [16, Definition 8.3.7] and such slices are actually Cauchy hypersurfaces.

From the physical viewpoint, the existence of a global splitting allows the observers in $Z$ to agree in a kind of average time $t$, or compromise time. Notice that the observers in $Z$ are proper time sinchronizable if and only if $\alpha \equiv 1$. Equivalently, when $M$ is simply connected, the observers in $Z$ are proper time sinchronizable if and only if $Z$ is irrotational and its observers are free falling.

From the mathematical viewpoint, it seems reasonable to think that the geometry of a standard static spacetime $(M, g)$ admitting more than one splitting should be subject to some restrictions, since it should admit some special kind of symmetries. This is the case of the Lorentz-Minkowski spacetime or, more generally, the case of Lorentz manifolds $(M, g)$ admitting a splitting as

$$
M=\mathbb{L}^{2} \times N, \quad g=\alpha^{2} g_{L}+g_{N},
$$

where $g_{L}$ is the usual metric in the Lorentz-Minkowski plane $\mathbb{L}^{2},\left(N, g_{N}\right)$ a complete Riemannian manifold and $\alpha$ a positive smooth function on $N$. A complete study of the geometric structure of the standard static spacetimes admitting several splittings can be found in [10].

Standard static spacetimes appear naturally in the context of the singularity theorems. As it is well known, the classical singularity theorems states, under some mild hypothesis including the timelike convergence condition, the existence of incomplete geodesics. Recall that a spacetime $(M, g)$ satisfies the timelike convergence condition when $\operatorname{Ric}(v, v) \geq 0$ for all timelike vector $v$, where Ric denotes the Ricci tensor of $(M, g)$. It is normally argued that the timelike convergence condition is the mathematical translation that gravity, on average, attracts [16]. This yielded Yau in the early 1980's to propose a "rigid singularity theorem", which was later stated by Bartnik as the following conjecture: any timelike geodesically complete spacetime $(M, g)$ of dimension greater than two, containing a compact Cauchy surface and satisfying the timelike convergence condition should split as in (2) with $\alpha \equiv 1$ (see [3]; and also $[8,18]$ for recent developments). This idea led Yau to also state the problem of obtaining the Lorentz analogue of the well-known (Riemannian) splitting theorem obtained by Cheeger-Gromoll [9], under the additional assumption of the existence of a complete timelike line (see for instance [4] and the references therein for a further analysis). So, it is natural to deal with a global splitting such that $I=\mathbb{R}$.

Therefore, the splitting provides essential information for both, the geometry and the physical properties of the model. Thus, the question on the uniqueness of a global standard static splitting arises naturally. Notice that if there is only one possible standard static splitting, then such a splitting singles a distinguished timelike direction out (represented by $\partial_{t}$ ). In this case, $Z$ is the only complete and integrable observer field, whose observers perceive its commom physical space as static. 
The first studies about uniqueness of standard static splittings are due to Sánchez-Senovilla [17] and later by Aledo-Romero-Rubio [2] for spatially closed spacetimes, i.e., spacetime models admitting a compact spacelike hypersurface (which happens if and only if $\Sigma$ is a compact Riemannian manifold [2, Section 3]).

Our aim in this paper is to provide a different and general framework for the study of the uniqueness of decomposition for standard static spacetimes including, not only the results obtained by Sánchez-Senovilla and Aledo-RomeroRubio; but also new results for the general case of a spatially open standard static spacetime, i.e., when the base is a complete, but non-necessarily compact, Riemannian manifold.

The paper is organized as follows. In Section 2 we introduce the well-known standard conformastationary spacetimes and some related concepts, as well as some known splitting results for such spaces, both from a topological and from a metric level. Observe that a standard static spacetime is a particular case of such spacetimes. In Section 3 we review Sánchez-Senovilla and AledoRomero-Rubios's previous results about the uniqueness of global splittings in the spatially closed case.

Finally, Section 4 is devoted to present several novel results. Specifically, we introduce a new global approach for the study of standard static splittings. To begin with, as a consequence of the strong maximum principle for elliptic partial differential equations we get a uniqueness result for maximal Cauchy hypersurfaces in the spatially closed case, Theorem 3. As a consequence, we get an alternative proof for the uniqueness results in this case. However, we realize that we do not need to ask the compactness assumption in order to get unicity, but just to observe that the projection of the timelike Killing vector field $K$ onto some of the spacelike slices $\Sigma_{t_{0}}$ vanishes somewhere, Theorem 4 . Thanks to that, we obtain new uniqueness results under assumptions on the sectional curvature of $(\Sigma, h)$. Such conditions are satisfied, for instance, for any ruled surface in $\mathbb{R}^{3}$, see Corollary 2. Our last result, Theorem 4.6, shows a different situation where Theorem 4.3 can be applied. Specifically, we prove uniqueness in the case where $M$ is a 3 -dimensional spacetime admitting a standard static splitting, and a certain condition on the orbits of a Killing vector field is satisfied.

\section{On standard stationary splittings}

Let $(M, g)$ be a chronological spacetime with a global complete timelike conformal Killing vector field $K$ defined on it. As it was proved by Harris on [11], such a vector field allows us to obtain a topological and differentiable global splitting $M=\mathbb{R} \times Q$, where $Q$ represents the space of integral curves of $K$ endowed with a natural manifold topology.

Later Javaloyes and Sánchez [12] characterized when such a splitting is extensible to the metric level, being expressable as a standard conformasta- 
tionary spacetime, that is, a spacetime $(M, g)$ such that

$$
M=\mathbb{R} \times \Sigma, \quad g=\Omega(s, x)\left(-\alpha^{2}(x) d s^{2}+\omega \otimes d s+d s \otimes \omega+h\right),
$$

where $\Sigma$ is a spacelike global section of $Q$, and so, it is intersected once for every integral curve of $K, \Omega$ and $\alpha$ are positive smooth functions on $M$ and $\Sigma$ respectively, and $\omega$ and $h$ are a 1 -form and a Riemannian metric on $\Sigma$. Their main result was the following:

Theorem 1 [12, Theorem 1.2] Let $(M, g)$ be a spacetime admitting a complete timelike conformal Killing vector field $K$. Then, $(M, g)$ can be expressed as a standard conformastationary splitting if, and only if, $(M, g)$ is distinguishing. Moreover, in this case $(M, g)$ is causally continuous.

Observe that conformastationary models include some well-known classical models. For instance, if $\Omega=1$ (or it is independent of $s$ ), then (4) becomes a standard stationary spacetime. It is quite straightforward that, in this situation, $K$ is in fact a Killing vector field.

When $\omega=0$, then (4) becomes a standard conformastatic metric. In this case, $K^{\perp}$ is an integrable distribution with $\Sigma$ one of its leaves. If, in addition, $\Omega=1$, we recover the standard static metric.

Remark 1 It is quite straightforward (see for instance [12, Lemma 2.1]) that if $K$ is a timelike conformal Killing vector field with respect to $g$, then it is Killing with respect to the conformal metric $g^{*}=-\frac{g}{g(K, K)}$. For the scope of this paper, we will restrict our studies to spacetimes admitting global timelike Killing vectors.

Definition 1 Let $(M, g)$ be an $(n+1)$-dimensional spacetime, $K$ a globally defined timelike conformal Killing vector field on $M$, and $\Sigma$ an $n$-dimensional hypersurface. We will say that $(M, g)$ admits a standard stationary splitting $(K, \Sigma)$ if it admits a splitting as in (4) with $\Omega=1$ and $\partial_{s}=K$, and so, $K$ is in fact a Killing vector field. If in addition $\omega=0$, then we will say that $(M, g)$ admits a standard static splitting $(K, \Sigma)$.

It is interesting to note that a splitting as a standard stationary spacetime is not unique. In fact, it depends on several factors, but mainly on the section of $Q$ we have considered. Nevertheless, once we have fixed one splitting, the set of all the possible splittings as standard stationary spacetimes related to the same Killing vector field is identifiable with a subspace of the space of smooth functions on $\Sigma$. To prove this fact, let us consider $K$ a globally defined timelike Killing vector field on $M$ and $\Sigma$ a spacelike hypersurface, so that $M$ admits a standard stationary splitting $(K, \Sigma)$. Consider now another spacelike hypersurface $\tilde{\Sigma}$ in $M$ satisfying that any integral curve on $K$ intersects it once. Observe that this fact is satisfied whenever $\Sigma$ is complete and simply connected.

Under the previous conditions, we can define the function

$$
f: \Sigma \rightarrow \mathbb{R}, \quad \text { such that } \quad(f(x), x) \in \tilde{\Sigma} \quad \text { for all } x \in \Sigma .
$$


Intuitively, the function $f$ determines the height between the points in $\Sigma \equiv$ $\{0\} \times \Sigma$ and $\tilde{\Sigma}$ when we move along the integral curves of $K$. Then, such a function defines a diffeomorphism

$$
\begin{gathered}
\Psi: \mathbb{R} \times \Sigma \rightarrow \quad \mathbb{R} \times \Sigma \\
(s, x) \rightarrow(s+f(x), x)
\end{gathered}
$$

which becomes an isometry if we endow the latter with the metric

$$
g=-\alpha^{2} d t^{2}+\tilde{\omega} \otimes d t+d t \otimes \tilde{\omega}+\tilde{h}
$$

where $\tilde{\omega}=\omega+\alpha^{2} d f$ and $\tilde{h}=h-\omega \otimes d f-d f \otimes \omega-\alpha^{2} d f^{2}$ (observe that we have just considered the variable change $t=s+f(x))$.

Finally, observe that when the original splitting $(K, \Sigma)$ is, in fact, static, it follows in particular that $\tilde{h}=h-\alpha^{2} d f^{2}$. Then, in order to ensure the spatial character of $\tilde{\Sigma}$, the function $f$ should satisfy that

$$
\|d f\|_{h}^{2}<\frac{1}{\alpha^{2}}, \quad \text { where }\|\cdot\|_{h} \text { denote the norm related to } h
$$

\section{Previous approaches for uniqueness results in the spatially closed} case

Let us make a review on the main results about the uniqueness of standard static splittings in spatially closed spacetimes that we can find on the literature. We will review [17] and [2], where the authors obtain uniqueness results for standard static spacetimes with a compact spacelike base.

\subsection{Sánchez and Senovilla's approach}

In [17] Sánchez and Senovilla proved the uniqueness of the splitting by means of an analysis of the properties of embedded totally geodesic manifolds on standard static models. Concretely, let $N$ be an embedded totally geodesic submanifold of a standard static spacetime $M=\mathbb{R} \times \Sigma$, and let us denote by $\tau$ the restriction of the time function on $M$ to $N$, that is, $\tau=\left.\pi_{\mathbb{R}}\right|_{N}$, (observe that here $\tau=-f$ on the approach in Section 2). Then, in [17, Proposition 2] they showed that

$$
\int_{N} \alpha^{2}\|d \tau\|^{2}=0
$$

deducing that $\tau \equiv t_{0}$ should be constant. Consequently, $N$ is contained in $\Sigma_{t_{0}}=\left\{t_{0}\right\} \times \Sigma$. The result strongly relies on the use of the divergence theorem, and seems to be extensible whenever we ensure that such a theorem is applicable. In fact, this idea led the authors to claim that a non-compact analogous of this result can be achieved if $\alpha$ satisfies some appropriate decaying properties. 
However, the situation seems to be a little more technical as it could be expected, and conditions on $\alpha$ seems to be not enough by themselves. In fact, in order to ensure that $N$ is spacelike, the relation (6) between $\alpha$ and $\tau=-f$ should hold. Let us assume, for instance, that $\alpha^{2} \sim O\left(r^{-l}\right)$, i.e., the decay of $\alpha^{2}$ is of order $-l$, then from (6) it is possible for $d f$ to satisfy $\|d f\|_{h}^{2} \sim O\left(r^{l}\right)$. Hence, the product inside (7) would behave as a $O(1)$. Therefore, if $N$ has infinite volume, such an integral could be infinity.

\subsection{Aledo, Romero and Rubio's approach}

The approach followed on [2] is radically different. Given $(K, \Sigma)$ and $(\tilde{K}, \tilde{\Sigma})$ two different standard static splittings with compact bases, Aledo-RomeroRubio's approach makes a comparison between the volumes of $\Sigma$ and $\tilde{\Sigma}$.

Their proof relies on [2, Proposition 1], where they compute the volume of one of the spacelike bases in terms of the volume element of the second one and the hyperbolic angle between both hypersurfaces. It is worth mentioning that the proof of such a result only requires that both $\Sigma$ and $\tilde{\Sigma}$ have finite volume, so it can be stated in the following more general way:

Lemma 1 Let $(M, g)$ be a standard static spacetime admitting a standard static splitting $(K, \Sigma)$ with $\Sigma$ a complete, orientable and simply connected $n$-Riemannian manifold with finite volume. If $\tilde{\Sigma}$ is a complete spacelike hypersurface in $M$, then $\tilde{\Sigma}$ must have finite volume.

Proof Let us recall the main ideas of the proof for the sake of completeness. From the assumptions of the lemma it follows easily that $\tilde{\Sigma}$ must be orientable. Let us denote by $\theta^{\tilde{\Sigma}}$ the Riemannian volume element of $\tilde{\Sigma}$. Following the same argument as in [2, Proposition 1] we have that

$$
\operatorname{vol}(\Sigma)=\int_{\tilde{\Sigma}} \cosh \varphi \theta^{\tilde{\Sigma}}
$$

Finally, the proof follows by applying the mean value theorem for the integral, via an exhaustion procedure.

Then, following the same arguments as in [2, Section 4] we get the following uniqueness result,

Theorem 2 Let $(M, g)$ be a spacetime admitting a standard static splitting $(K, \Sigma)$ with $\Sigma$ a complete and simply connected $n$-Riemannian manifold, with finite volume. Then, every irrotational timelike Killing vector field $\tilde{K}$ on $M$ must be proportional to $K$.

\section{On the uniqueness result: A global approach}

As far as we know, up to the moment the only known uniqueness result in the general case is due to Gutiérrez and Olea. Specifically, in [10, Theorem 
5.7] they show that the uniqueness of the splitting is guaranteed whenever a certain condition on the lightlike sectional curvature is satisfied. They also give an alternative proof of the uniqueness of the decomposition in the spatially closed situation.

Our aim in this section is to present a different approach for the uniqueness problem for general standard static splittings. Although we are able to recover again the spatially closed case, our techniques are also applicable to a more general context.

Let us begin by considering $(M, g)$ a standard static spacetime as in (2), $\tilde{\Sigma}$ an immersed spacelike hypersurface on $M$, and the time function $\tau: \tilde{\Sigma} \rightarrow \mathbb{R}$ as defined in Section 2. Since $\tau=\left.\pi_{\mathbb{R}}\right|_{\tilde{\Sigma}}$, it yields $\tilde{\nabla} \tau=\left(\bar{\nabla} \pi_{\mathbb{R}}\right)_{\tilde{\Sigma}}$, $\tilde{\nabla}$ and $\bar{\nabla}$ being the gradient operators in $\tilde{\Sigma}$ and $M$, respectively, and $V_{\tilde{\Sigma}}$ the orthogonal projection of $V$ onto $\tilde{\Sigma}$ for any $V \in \mathfrak{X}(M)$. Therefore, it follows from the metric on (2) that

$$
-\alpha^{2} \tilde{\nabla} \tau=\left(\partial_{t}\right)_{\tilde{\Sigma}} .
$$

Recalling that $\partial_{t}$ is Killing, together with the Gauss formula, we get

$$
g\left(\tilde{\nabla}_{X}\left(\partial_{t}\right)_{\tilde{\Sigma}}, X\right)+g\left(N, \partial_{t}\right) g(A X, X)=0,
$$

for every $X \in \mathfrak{X}(\tilde{\Sigma})$, where $\tilde{\nabla}$ stands for the Levi-Civita connection in $\tilde{\Sigma}, N$ the future-pointing unitary normal field to $\tilde{\Sigma}$ in $M$, and $A$ its related Weingarten operator.

Considering now $\left\{E_{i}\right\}$ an orthonormal frame of $\tilde{\Sigma}$ around a point $p$ with respect to the the induced metric, we get

$$
\begin{aligned}
\tilde{\triangle} \tau & =-\sum_{i=1}^{n} g\left(\tilde{\nabla}_{E_{i}}\left(\frac{1}{\alpha^{2}}\left(\partial_{t}\right)_{\tilde{\Sigma}}\right), E_{i}\right) \\
& =-\sum_{i=1}^{n} E_{i}\left(\frac{1}{\alpha^{2}}\right) g\left(\left(\partial_{t}\right)_{\tilde{\Sigma}}, E_{i}\right)-\frac{1}{\alpha^{2}}\left(\sum_{i=1}^{n} g\left(\tilde{\nabla}_{E_{i}}\left(\partial_{t}\right)_{\tilde{\Sigma}}, E_{i}\right)\right),
\end{aligned}
$$

$\tilde{\triangle}$ being the Laplacian operator in $\tilde{\Sigma}$, and recalling (9),

$$
\tilde{\triangle} \tau=-\frac{2}{\alpha} g(\tilde{\nabla} \alpha, \tilde{\nabla} \tau)+n H \frac{\cosh (\theta)}{\alpha},
$$

where $H$ is the mean curvature of $\tilde{\Sigma}$ with respect to $N$ and $\theta$ the hyperbolic angle between the timelike vector fields $N$ and $\partial_{t}$. Let us observe that if $\tilde{\Sigma}$ is maximal, then the previous equation can be expressed as

$$
(\mathcal{L}(\tau):=) \alpha \tilde{\triangle} \tau+2 \tilde{h}(\tilde{\nabla} \alpha, \tilde{\nabla} \tau)=0,
$$

$\tilde{h}$ being the metric induced on $\tilde{\Sigma}$ from the metric $g$, which is a linear elliptic partial differential equation. From (11), we can easily prove the following result: 
Theorem 3 Let $(M, g)$ be a spatially closed standard static spacetime as in (2). Then, there exists no other maximal Cauchy hypersurface but the slices $\Sigma_{t_{0}}$.

Proof First observe that any Cauchy hypersurface $\tilde{\Sigma}$ on $M$ should be compact, as any two Cauchy hypersurfaces are homeomorphic. Now recall that the time function $\tau: \tilde{\Sigma} \rightarrow \mathbb{R}$ satisfies the elliptic differential equation (11). As $\tilde{\Sigma}$ is compact, the strong maximum principle (see for instance [14, Theorem 2.9]) ensures that $\tau$ is necessarily constant. Thus, $\tilde{\Sigma}=\Sigma_{t_{0}}$ for a certain constant $t_{0}$.

Corollary 1 A spacetime $(M, g)$ admitting a compact Cauchy hypersurface admits at most one standard static splitting.

Proof Assume that $(M, g)$ admits a standard static splitting $(K, \Sigma)$, so it can be expressed as in (2) with $\partial_{t}=K$. Taking into account the assumption of the corollary, $\Sigma$ is necessarily a compact Cauchy hypersurface. Now observe that, if there exists another standard static splitting $(\tilde{K}, \tilde{\Sigma})$, then $\tilde{\Sigma}$ should be maximal. Finally, Theorem 3 ensures that $\tilde{\Sigma}=\Sigma_{t_{0}}$, for some constant $t_{0}$, concluding that both $(K, \Sigma)$ and $(\tilde{K}, \tilde{\Sigma})$ define the same splitting.

Previous results only make special use of the fact that $\tilde{\Sigma}$ is maximal. However, the existence of another standard static splitting $(\tilde{K}, \tilde{\Sigma})$ will require not just $\tilde{\Sigma}$ to be a maximal hypersurface, but a totally geodesic one. As we are going to see, this stronger condition derives on stronger restrictions for the existence of several splittings.

Following this idea, let $(M, g)$ be a spacetime admitting a standard static splitting $(K, \Sigma)$, and let $\tilde{K}$ be another timelike Killing vector field. From now on, given any point $p=(t, x) \in M$ let $\left.\tilde{K}_{\Sigma_{t}}\right|_{p}$ be the horizontal projection of $\left.\tilde{K}\right|_{p}$ in the sense of [15, Chapter 7], i.e., its orthogonal projection onto $T_{p} \Sigma_{t}$. Observe that, if $\left.\tilde{K}_{\Sigma_{t}}\right|_{p}=0$ for some point $p=(t, x) \in M$, both $\tilde{K}$ and $K$ are proportional at $p$, and so, they have the same orthogonal vector subspace. Then, we can obtain the following result,

Theorem 4 Let $(M, g)$ be a complete spacetime admitting two standard static splittings $(K, \Sigma)$ and $(\tilde{K}, \tilde{\Sigma})$. If $\tilde{K}_{\Sigma_{t_{0}}}$ has some zero on $\Sigma_{t_{0}}$ for some $t_{0} \in \mathbb{R}$, then both splittings coincide.

Proof $\Sigma$ and $\tilde{\Sigma}$ are totally geodesic hypersurfaces in $M$ whose tangent spaces are orthogonal to $K$ and $\tilde{K}$ respectively, so they are $\Sigma_{t}$ and $\tilde{\Sigma}_{s}$ for any $t, s \in \mathbb{R}$. If there exists $t_{0}$ where $\left.\tilde{K}_{\Sigma_{t_{0}}}\right|_{p}=0$ for some point $p$, then both Killing vector fields have the same orthogonal vector subspace at $p$, i.e., $T_{p} \Sigma_{t_{0}}=T_{p} \tilde{\Sigma}_{s_{0}}$ for some $s_{0} \in \mathbb{R}$. As both $\Sigma_{t_{0}}$ and $\tilde{\Sigma}_{s_{0}}$ are complete totally geodesic manifolds, we deduce that $\Sigma_{t_{0}}=\tilde{\Sigma}_{s_{0}}$. In conclusion, $(K, \Sigma)$ and $(\tilde{K}, \tilde{\Sigma})$ determine the same splitting.

As a consequence of the previous result, we can obtain an alternative proof of Corollary 1. For this, just observe that, with a similar argument as in (8), 
but interchanging the roles of $\partial_{t}$ and $\tilde{K}$, it follows that for any $t \in \mathbb{R}$ it holds

$$
\tilde{K}_{\Sigma_{t}}=-\tilde{\alpha}^{2} \nabla f
$$

for certain functions $\tilde{\alpha}, f: \Sigma_{t} \rightarrow \mathbb{R}$, where $\nabla$ is the gradient operator in $\Sigma_{t}$. As the function $f$ is defined over the compact set $\Sigma_{t}$, it should have a critical point, and hence, $\tilde{K}_{\Sigma_{t}}$ should have some zero from equation (12).

However, this approach allows other possible uniqueness results. In fact, recalling a classical result (see for instance [1, Theorem 5.4]), the orthogonal projection of a Killing vector field $\tilde{K}$ on $\Sigma$ is also a Killing vector field on $\Sigma$ with respect to the induced (Riemannian) metric. Let us denote by Iso $(M)$ the set of isometries on $M$, and with $\operatorname{Iso}_{0}(M)$ its connected component containing the identity. As it is well known, the flow of a (globally defined) Killing vector field naturally defines a one-parameter family of isometries living on $\operatorname{Iso}_{0}(\Sigma)$, and fixed points of this family are related with zeroes of the Killing vector field. In case that $\operatorname{Iso}_{0}(M)$ is a compact group (for instance, if $\Sigma$ cannot be expressed as a product $\mathbb{R} \times Q$ ), we can obtain uniqueness results in the following way:

Corollary 2 Let $(M, g)$ be a complete and simply connected spacetime admitting a standard static splitting $(K, \Sigma)$, assume that $\operatorname{Iso}_{0}(\Sigma)$ is a compact group, where $\Sigma$ is endowed with the induced Riemannian metric $h$, and that one of the following properties holds:

(i) $(\Sigma, h)$ has non-positive sectional curvature or,

(ii) $(\Sigma, h)$ has positive sectional curvature.

Then, previous standard static splitting is unique.

Proof The proof follows by showing that $\operatorname{Iso}_{0}(\Sigma)$ necessarily has a fixed point, which follows by Cartan's fixed point theorem if we assume (i) (see for instance [7, Theorem 4.0.1]), and from [20, Section 3] if we assume (ii).

Let us observe that there exists a big variety of Riemannian manifolds $\Sigma$ such that $\operatorname{Iso}_{0}(\Sigma)$ is compact and either (i) or (ii) of Proposition 2 is satisfied. In fact, those examples exist even in the simplest case where $\Sigma$ is an embedded surface in $\mathbb{R}^{3}$.

On the one hand, it is well-known that any ruled surface in $\mathbb{R}^{3}$ has nonpositive Gaussian curvature. Therefore, we can consider any simply connected ruled surface in $\mathbb{R}^{3}$ with compact isometry group, as it is the hyperbolic paraboloid. On the other hand, any simply connected rotational surface in $\mathbb{R}^{3}$, generated by a strictly convex curve, has positive Gaussian curvature and compact isometry group. In fact, its isometry group coincides with $S O(2)$. In this family we can consider, for instance, one of the two connected components of the two-sheeted hyperboloid.

Let us note that previous uniqueness result is obtained under global conditions on $(\Sigma, h)$ which ensure that all Killing vector fields on $\Sigma$ have a common zero. However, our next uniqueness result will focus on conditions for concrete Killing vector fields. The result will follows from the following technical lemma for 2-Riemannian manifolds 
Lemma 2 Let $(\Sigma, h)$ be a complete, non-compact, simply connected and orientable 2-Riemannian manifold. If a Killing vector field $K$ has at least a closed orbit, then there exists a point $x$ where $\left.K\right|_{x}=0$.

Proof Let $K$ be a Killing vector field with at least a closed orbit, and assume by contradiction that $K$ has no zeroes. Thus, the global flow of $K$ defines a 1-foliation $\mathcal{F}$ on $\Sigma$. Making use of the uniformization theorem (see, [6, Chapter IV]), we know that the smooth surface $\Sigma$ is diffeomorphic to the plane $\mathbb{R}^{2}$.

The Kamke's theorem (see [5], [13]) is called to assure the existence of a smooth map $f: U \longrightarrow \mathbb{R}$, which is constant on the leaves of $\left.\mathcal{F}\right|_{U}$ and such that $d f \neq 0$ at each point of $U$, where $U$ is a bounded subset of $\Sigma$ containing the closed orbit $\mathcal{O}$. Let $\sigma:[a, b] \subset \mathbb{R} \longrightarrow U$ be a transverse smooth curve to $\left.\mathcal{F}\right|_{U}$ satisfying $\sigma(a), \sigma(b) \in \mathcal{O}$ and $\sigma(a) \neq \sigma(b)$. Now, consider the function $f \circ \sigma$, via the Rolle's theorem we know that there exists $s_{0} \in[a, b]$, such that $(f \circ \sigma)^{\prime}\left(s_{0}\right)=0$ and as a consequence $\left.d f\right|_{\sigma\left(s_{0}\right)}=0$, so we get a contradiction.

As a consequence of previous Riemannian result,

Theorem 5 Let $(M, g)$ be a 3-dimensional spacetime admitting a standard static splitting $(K, \Sigma), \Sigma$ being a complete, non-compact and simply connected 2-Riemannian manifold. If the horizontal projection onto some slice $\Sigma_{t_{0}}$ of any other timelike Killing vector field $\tilde{K}$ in $M$ has at least a closed orbit, then the splitting is unique.

Proof Let $(\tilde{K}, \tilde{\Sigma})$ be a standard static splitting of $(M, g)$. From hypothesis, the Killing vector field $\tilde{K}_{\Sigma_{t_{0}}}$ has a closed orbit on $\Sigma_{t_{0}}$, and so, a zero from Lemma 2 . Then Theorem 4 ensures that both $(K, \Sigma)$ and $(\tilde{K}, \tilde{\Sigma})$ coincide.

\section{References}

1. Transformation Groups in Differential Geometry — Shoshichi Kobayashi — Springer

2. Aledo, J.A., Romero, A., Rubio, R.M.: The existence and uniqueness of standard static splitting. Classical and Quantum Gravity 32(10), 105004 (2015). DOI 10.1088/0264-9381/32/10/105004

3. Bartnik, R.: Remarks on cosmological spacetimes and constant mean curvature surfaces. Communications in Mathematical Physics 117(4), 615-624 (1988)

4. Beem, J.K., Ehrlich, P.E., Easley, K.L.: Global Lorentzian Geometry, Pure and Applied Mathematics, vol. 202. Marcel Dekker, New York (1996)

5. Camacho, C., Neto, A.L.: Geometric Theory of Foliations. Birkhäuser Basel (1985)

6. Farkas, H.M., Kra, I.: Riemann Surfaces, 2 edn. Graduate Texts in Mathematics. Springer-Verlag, New York (1992)

7. Farmakis, I., Moskowitz, M.: Fixed Point Theorems and Their Applications. World Scientific Publishing Company, Hackensack, New Jersey (2013)

8. Galloway, G.J., Vega, C.: Achronal Limits, Lorentzian Spheres, and Splitting. Annales Henri Poincaré 15(11), 2241-2279 (2014). DOI 10.1007/s00023-013-0305-1

9. Gromoll, D., Meyer, W.: On Complete Open Manifolds of Positive Curvature. Annals of Mathematics 90(1), 75-90 (1969). DOI 10.2307/1970682

10. Gutiérrez, M., Olea, B.: Uniqueness of static decompositions. Annals of Global Analysis and Geometry 39(1), 13-26 (2010). DOI 10.1007/s10455-010-9222-4. URL http://dx . doi.org/10.1007/s10455-010-9222-4 
11. Harris, S.: Conformally stationary spacetimes. Classical and Quantum Gravity 9(7), $1823(1992)$

12. Javaloyes, M.A., Sánchez, M.: A note on the existence of standard splittings for conformally stationary spacetimes. Classical and Quantum Gravity 25(16), 168001 (2008)

13. Kamke, E.: Über die partielle differentialgleichung $f(x, y) \frac{\partial z}{\partial x}+g(x, y) \frac{\partial z}{\partial x}=h(x, y)$. Mathematische Zeitschrift 41(1), 56-66 (1936). DOI 10.1007/BF01180405. URL https: //doi.org/10.1007/BF01180405

14. Kazdan, J.L.: Some applications of partial differential equations to problems in geometry. J.S. Kazdan (1983)

15. O'Neill, B.: Semi-Riemannian Geometry With Applications to Relativity, 103, Volume 103 (Pure and Applied Mathematics). Academic Press (1983)

16. Sachs, R.K., Wu, H.H.: General relativity for mathematicians. Graduate Texts in Mathematics (1977). DOI 10.1007/978-1-4612-9903-5. URL http://dx.doi.org/10.1007/ 978-1-4612-9903-5

17. Sánchez, M., Senovilla, J.M.M.: A note on the uniqueness of global static decompositions. Classical and Quantum Gravity 24(23), 6121 (2007). DOI 10.1088/0264-9381/ $24 / 23 / \mathrm{N} 01$

18. Sharifzadeh, M., Bahrampour, Y.: Some Results about the Level Sets of Lorentzian Busemann Function and Bartnik's Conjecture. Communications in Mathematical Physics 286(1), 389-398 (2009). DOI 10.1007/s00220-008-0609-z

19. Warner, F.W.: Foundations of Differentiable Manifolds and Lie Groups, Graduate Texts in Mathematics, vol. 94. Springer-Verlag New York (1983)

20. Yau, S.T.: Remarks on the group of isometries of a Riemannian manifold. Topology 16(3), 239-247 (1977). DOI 10.1016/0040-9383(77)90004-0 